\section{Turing patterns in fish skin?}

SIR - Kondo and Asai ${ }^{1}$ interpret observations on the time evolution of skin patterns of the angelfish (Pomacanthus) as the first instance of a Turing (reaction-diffusion) pattern in biology. But we believe that reaction-diffusion systems per se cannot provide a mechanistic basis for one of the main patterns reported in ref. 1.

Reaction-diffusion systems are characterized by an intrinsic spatial wavelength of the self-organized concentration pattern, that is, the distance between adjacent peaks of chemical concentrations is determined solely by the system parameters (kinetic constants and diffusion coefficients). Although on a two-dimensional domain such as the fish skin, several equidistant geometrical arrangements of the concentration peaks are possible, the nonlinear terms of the reaction dynamics

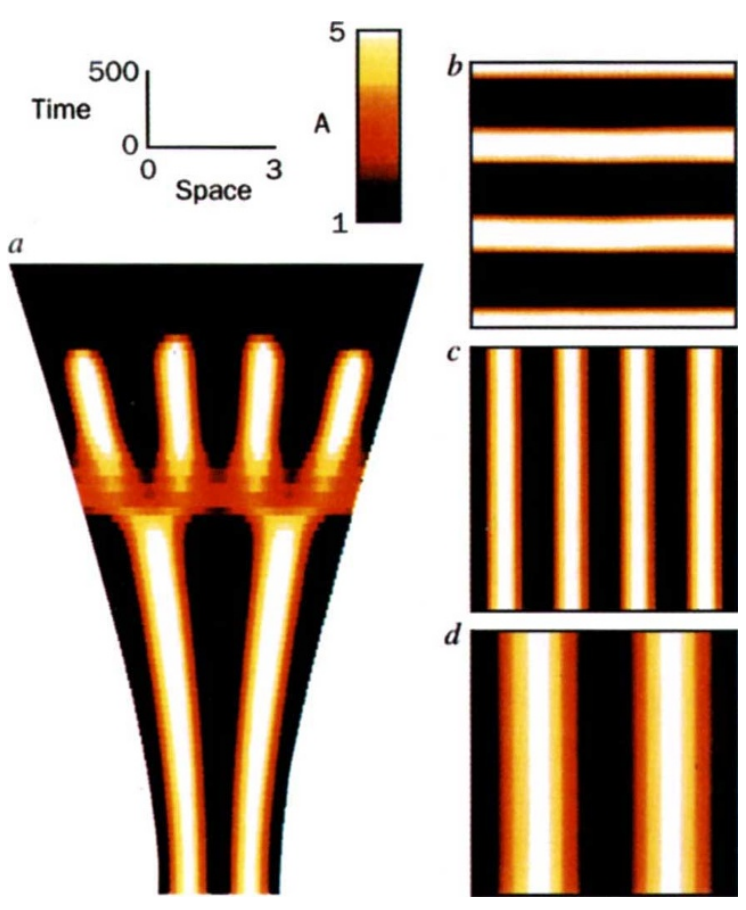

Behaviour of the Turing system proposed in ref. 1 on a growing square domain (with the signs of the diffusion terms corrected). $a$, Concentration plot of $A$ in a horizontal cross-section of the domain; time increases from bottom to top (see separate scales). The 4-stripe pattern produced by the first perioddoubling is unstable, rearranges into a 3-stripe pattern perpendicular to the original pattern, and the stripe contours terminate. $b-d$, Snapshots of the stripe patterns correspond ing to a (domains scaled to same size): $b$, initial 2-stripe pattern $(t=500)$; $c$, after period-doubling $(t=3,000)$; and $d$, after rearrangement into 3 stripes $(2+2$ half-stripes, $t=4,000$ corresponding to the dark region in a). Simulations: equations scaled to the form $\partial u / \partial t=s^{2} f(u)+D \nabla^{2} u$, and solved with a standard ADI scheme on a fixed domain (mesh size 0.2, time step 0.05) with zero flux boundary conditions; increase in $s$ is equivalent to increase in (domain length) ${ }^{2}$, here $s(t)=\sqrt{\left(0.15+10^{-7} t^{2}\right)}$. Patterning sequence is sensitive to the speed of domain growth and for faster growth rates the transitions become less controlled; we found transitions from 2 stripes to higher modes ( 5 stripes and more) with subsequent rearrangements. form a new stripe pattern enlarged by one stripe. This behaviour does not depend on the aspect ratio of the domain; we have found complete perpendicular rearrangement of pattern even on very narrow (quasi-one-dimensional) domains. Thus, the patterning dynamics must involve an interplay of the mechanism that sets the distance between adjacent stripes and some form of 'memory' that conserves the location of old stripes. The 'memory' could be provided by pigment cells forming stable aggregations ${ }^{4}$. More specific quantitative models based on experimentally implicated mechanisms are needed to formulate testable predictions on the origin of the dynamic Pomacanthus skin patterns.

\section{Thomas Höfer}

Philip K. Maini

Centre for Mathematical Biology,

Mathematical Institute,

University of Oxford, 24-29 St Giles', Oxford OX1 3LB, UK

KONDO AND ASAI REPLY - With respect to Höfer and Maini's first criticism, we agree that many pattern-forming systems can explain the phenomenon we observed. These models have in common a set of interactions involving local activation/ lateral inhibition coupled with the appropriate nonlinearities 5 . The most important message of our report ${ }^{1}$ is that a dynamical mechanism like Turing's is viable for the fish patterns. It should therefore be possible to identify the real molecular mechanism by experiments. Of course, at present the details of the fish-patterning mechanism are unknown, and will not be understood until experiments are done.

Second, Höfer and Maini claim that a two-dimensional simulation of the $P$. semicirculatus pattern is more realistic than the one-dimensional simulation in our paper. This is by no means clear. All the stripe lines of $P$. semicirculatus are perpendicular to the body axis and there are no branch points. These features suggest the presence of a directional preference forcing the stripes to run in the same direction. A one-dimensional simulation captures some of the character of this system better than does an isotropic two-dimensional simulation.

\section{Shigeru Kondo}

Kyoto University Centre for Molecular

Biology and Genetics,

Shogoin-Kawaharacho 53, Sakyo-ku,

Kyoto 606, Japan

Rihito Asai

Kyoto University Seto Marine

Biological Laboratory,

Shirahama-cho, Nishimuro-gun,

Wakayama 649-22, Japan

1. Kondo, S. \& Asai, R. Nature 376, 765-768 (1995)

2. Cross, M. C. \& Hohenberg, P. C. Rev. mod. Phys. 65, 851-1112 (1993).

3. Murray, J. D. Mathematical Biology (Springer, Berlin, 1993).

4. Le Douarin, N. M. Curr. Topics dev. Biol. 16, 31-85 (1980).

5. Meinhardt, H. Nature 376, 722-723 (1995). 